\title{
Prevalência de depressão entre estudantes universitários
}

\author{
Depression prevalence among university students
}

\author{
Julio de Melo Cavestro e Fabio Lopes Rocha
}

\begin{abstract}
Resumo
Objetivo: Determinar e comparar os índices de depressão e risco de suicídio entre estudantes de medicina, fisioterapia e terapia ocupacional matriculados na Faculdade de Ciências Médicas de Minas Gerais (FCMMG) no ano de 2003. Métodos: Foram selecionados 342 estudantes através de amostragem por cotas. $O$ diagnóstico psiquiátrico foi realizado por meio do Mini International Neuropsychiatric Interview (MINI). A prevalência de transtornos depressivos foi estimada segundo curso, sexo e período letivo. Para comparação utilizou-se o teste qui-quadrado $\left(\chi^{2}\right)$ ou 0 teste exato de Fisher, com um nível de significância de $5 \%(p<0,05)$. Resultados: As taxas de prevalência de depressão entre os alunos foram medicina, 8,9\%; fisioterapia, 6,7\%; terapia ocupacional, $28,2 \%(p=0,002)$. As taxas de prevalência para o risco de suicídio entre os alunos foram medicina, 7,5\%; fisioterapia, 7,8\%; terapia ocupacional, $25,6 \%(p=0,005)$. Conclusão: As taxas de prevalência de depressão e do risco de suicídio entre os estudantes de terapia ocupacional foram significativamente mais elevadas quando em comparação com as observadas entre os de medicina e fisioterapia.
\end{abstract}

Palavras-chave: depressão, estudantes universitários.

\begin{abstract}
Objective: The aim of this study is to determine and compare the prevalence of depression and the suicide risk among the students from Minas Gerais Medical Science College (FCMMG), a private institution that offers medicine, physical therapy and occupational therapy courses. Methods: 342 students were selected according to a random sample by quota. The psychiatric diagnosis was taken by using the Mini International Neuropsychiatric Interview (MINI). The rates of the prevalence of depression and suicide risk were esteemed according to the course, gender and length in the course. For comparison, it was used the qui-square test $\left(\chi^{2}\right)$ or the exact Fisher's test, with a significance level of $5 \%(p<0.05)$. Results: The rates of the prevalence of depression obtained among the students were medicine, $8.9 \%$; physical therapy, $6.7 \%$; occupational therapy, $28.2 \%(p=0.002)$. The rates of prevalence of suicide risk among students were medicine, $7.5 \%$; physical therapy, $7.8 \%$; occupational therapy, $25.6 \%(p=0.005)$. Conclusion: The prevalence of depression and the suicide risk among the students of occupational therapy was significantly more elevated if compared to the rates obtained among the students of medicine and physical therapy.
\end{abstract}

Key words: depression, university students.

Faculdade de Ciências Médicas de Minas Gerais (FCMMG) (Cavestro JM)

Instituto de Previdência dos Servidores do Estado de Minas Gerais (IPSEMG) (Rocha FL) 


\section{Introdução}

Estima-se que $15 \%$ a $25 \%$ dos estudantes universitários apresentam algum tipo de transtorno psiquiátrico durante sua formação acadêmica (Adewuia et al., 2006; Eric et al., 1988; Giglio, 1975; Segall, 1966), notadamente transtornos depressivos e de ansiedade (Hahn e Ferraz, 1998; Millan et al., 1995; Niemi, 1984; Nucette, 1985). A maioria desses estudos foi realizada entre estudantes de medicina (Chan, 1992; Clark e Zeldow, 1988; Cordáz et al., 1988; Galli et al., 2001; Lloyd e Gartrell, 1984; Lloyd, 1983; Zoccolillo et al., 1986), portanto estima-se que a prevalência dos transtornos depressivos nessa população oscila entre $8 \%$ e $17 \%$.

Vários problemas metodológicos dificultam a comparação dos resultados dos diversos estudos, entre eles o levantamento de sintomas depressivos versus transtornos depressivos e a diversidade de instrumentos utilizados para a realização de diagnóstico e coleta de dados (Adewuia et al., 2006; Azi, 2003; Lloyd e Gartrell, 1984; Segal, 1966; Zoccolillo et al., 1986).

Recentemente, três estudos epidemiológicos utilizaram 0 Mini International Neuropsychiatric Interview (MINI) como instrumento diagnóstico. Um, realizado na Nigéria, envolveu estudantes universitários em geral (Adewuia et al., 2006), e a prevalência encontrada de transtorno depressivo maior foi de $8,3 \%$. Os outros dois estudaram especificamente alunos de medicina. Galli et al. (2001) reportaram prevalência de $24 \%$ de depressão maior e $15,6 \%$ de distimia em uma universidade peruana. No Brasil, a taxa de transtornos depressivos foi de $15,6 \%$ entre estudantes da Faculdade de Medicina de Salvador (Azi, 2003).

Um problema intimamente relacionado à depressão é o suicídio. Alguns estudos indicam elevado risco de suicídio entre os universitários em geral e, particularmente, entre os estudantes de medicina (Ross, 1973). Após os acidentes o suicídio seria a segunda causa mais comum de morte entre os estudantes de medicina (Rimmer et al., 1982).

Alguns autores acreditam que existam diferentes estressores ao longo de um curso universitário, dependendo do nível em que se encontre o aluno (início, meio ou final de curso) (Clark e Zeldow, 1988; Hahn e Ferraz, 1998; Lloyd e Gartrell, 1984; Millan et al., 1995; Millan e Barbedo, 1988; Pepitone-Arreola-Rockwell et al., 1981), e que esses fatores poderiam influenciar a prevalência de depressão entre os estudantes. No curso de medicina, os fatores estressores principais ocorreriam no início (volume de informações que o aluno passa a receber, mudanças nos métodos de estudo e carga horária exigida) e no final (insegurança com relação à própria competência e ao mercado de trabalho que começa a se descortinar) (Millan et al., 1995; Millan e Barbedo, 1988).

O objetivo deste estudo foi identificar e comparar a prevalência de transtornos depressivos e o risco de suicídio entre estudantes de medicina, fisioterapia e terapia ocupacional da Faculdade de Ciências Médicas de Minas Gerais (FCMMG).

\section{Métodos}

O estudo foi aprovado pelo Comitê de Ética em Pesquisa do Centro de Pós-graduação da Santa Casa de Misericórdia de Belo Horizonte, e os alunos forneceram consentimento escrito para sua participação.
No ano letivo de 2003, época da coleta de dados, havia 820 alunos na FCMMG, sendo 537 inscritos no curso de medicina, com duração de seis anos; 197 no curso de fisioterapia, com duração de cinco anos; e 86 alunos no curso de terapia ocupacional, com duração de quatro anos.

Foi utilizada a amostragem por cotas, sendo o curso de graduação o único estrato considerado. A amostra foi calculada a partir da prevalência estimada de transtornos psiquiátricos de 25\% (Azi, 2003; Chan, 1992; Eric et al., 1988; Nucette, 1985; Rimmer et al., 1982). Ao considerar a perda de $5 \%$, a estimativa foi de 302 o tamanho da amostra, correspondente a $35,2 \%$ do total. Foram entrevistados 342 alunos:

- $90(26,3 \%)$ do curso de fisioterapia;

- 213 da medicina $(62,3 \%)$;

- $39(11,4)$ da terapia ocupacional.

Estabeleceu-se a aleatorização da amostra a partir da divisão do total de 817 alunos (foram excluídos da pesquisa três alunos do sexo masculino do curso de terapia ocupacional) pelo tamanho calculado para a amostra (302), obtendo-se o resultado de 2,7. Após o arredondamento para 3, ficou estabelecido esse valor como intervalo entre um aluno e o próximo a ser entrevistado, de acordo com a posição na lista de chamada de cada turma, procedimento esse mantido para ambos os sexos. 0 aluno que serviria de ponto de partida na lista foi sorteado aleatoriamente, após todos terem sido numerados. Quando um aluno se recusava a participar do estudo, o subseqüente na lista era convidado a dar a sua colaboração à pesquisa.

O diagnóstico psiquiátrico e o risco de suicídio foram obtidos por meio do MINI, que é uma entrevista diagnóstica padronizada com duração de aproximadamente 15 minutos e que explora os principais transtornos psiquiátricos do eixo I da quarta revisão do Manual de Diagnóstico e Estatística das Perturbações Mentais (DSM-IV). Todas as entrevistas foram realizadas por um dos autores do estudo (Cavestro JM).

A análise estatística consistiu na estimativa da prevalência de transtornos depressivos segundo sexo, curso e ano. Para comparação utilizaram-se o teste qui-quadrado $\left(\chi^{2}\right)$ e/ou o teste exato de Fisher. Foram consideradas diferenças estatisticamente significativas aquelas cujo valor de $p$ foi $<0,05$. Com o objetivo de quantificar a razão de chances da prevalência do transtorno depressivo maior e do risco de suicídio, modelos de regressão logística foram testados.

\section{Resultados}

Dos 342 alunos entrevistados, $63 \%$ eram do sexo feminino e $27 \%$ do masculino. A idade média foi de aproximadamente 23 anos, variando de 18 a 36 anos. A maioria era da faixa etária entre 20 e 24 anos, representando 73,2\% da amostra. A idade média dos alunos de medicina foi superior à dos alunos dos demais cursos (Tabela 1).

A distribuição dos alunos por sexo entre os três cursos não foi uniforme. $O$ percentual de mulheres foi o seguinte:

- na terapia ocupacional, $100 \%$;

- na fisioterapia, $80 \%$;

- na medicina, 48,8\%.

A prevalência de episódios depressivos maior entre os alunos foi de $10,5 \%$, mas os do curso de terapia ocupacional 
apresentaram prevalência mais elevada $(28,2 \%)$ em comparação com os de medicina $(8,9 \%)$ e de fisioterapia $(6,7 \%)(p=$ $0,002)$. A diferença persistiu mesmo após ajustamento por sexo e idade. As taxas de prevalência para distimia foram de 2,8\% entre os alunos de medicina; $4,4 \%$ entre os de fisioterapia; e $2,6 \%$ entre os de terapia ocupacional, não havendo diferença significativa entre os três cursos (Tabela 1).

\section{Tabela 1. Distribuição de alunos de acordo com a idade e o curso freqüentado}

\begin{tabular}{lrcc}
\hline \multicolumn{1}{c}{ Curso } & Média \pm & Idade mínima & Máxima \\
\hline Fisioterapia & $21,85 \pm 2,57$ & 18,13 & 36,44 \\
Medicina & $23,09 \pm 2,26$ & 18,26 & 30,56 \\
Terapia & $21,02 \pm 1,94$ & 18,08 & 25,39 \\
ocupacional & & & \\
\hline
\end{tabular}

Valor de $p=0,005$.

O risco de suicídio foi observado em $9,6 \%$ dos alunos entrevistados, sendo $63,6 \%$ de risco baixo, $15,2 \%$ de risco médio e $21,2 \%$ de risco elevado. A prevalência foi significativamente maior entre os alunos de terapia ocupacional $(25,6 \%)$, em relação aos de medicina $(7,5 \%)$ e fisioterapia $(7,8 \%)(p=0,005)$. A diferença persistiu após ajustamento por sexo e idade.

Com a utilização de modelo de regressão logística, após ajuste por sexo e idade, estimou-se que a chance de ocorrer episódio depressivo maior entre as alunas da terapia ocupacional foi 3,6 vezes a chance de ocorrer entre as alunas de medicina e de fisioterapia. A chance do risco de suicídio entre as alunas de terapia ocupacional foi de 3,7 vezes a de ocorrer entre as alunas de medicina e fisioterapia.

Não foram observadas diferenças significativas na prevalência dos transtornos depressivos entre as etapas cursadas pelos alunos (início, meio e final de curso).

\section{Discussão}

A taxa de prevalência de 10,5\% para transtorno depressivo maior observada entre os estudantes da FCMMG encontra-se mais próxima do limite inferior daquelas observadas em diversos estudos desenvolvidos com estudantes universitários, cujos índices oscilam entre 8\% e 17\% (Chan, 1992; Clark e Zeldow, 1988; Eric et al., 1988; Galli et al., 2001; Rimmer et al., 1982; Zoccolillo et al., 1986). Entretanto, como já mencionado, a diversidade de métodos empregados em estudos semelhantes torna difícil a comparação de resultados. Entre as diferenças metodológicas, são particularmente relevantes os instrumentos utilizados para o diagnóstico (MINI, Minnesota Multiphasic Personality Inventory [MMPI], Beck Depression Inventory [BDI], Hopkins Symptom Checklist [HSCL], Standardized Psychiatric Interview [SPI], College Health Survey [CHS]), porém alguns avaliam a presença de sintomas, outros, de distúrbio psiquiátrico.

Em comparação com estudos semelhantes nos quais foram utilizados o MINI como instrumento para a coleta de dados, a taxa de prevalência de $10,5 \%$ para o transtorno de- pressivo maior observada entre os estudantes da FCMMG ficou próxima daquela encontrada entre os estudantes universitários nigerianos, que foi de $8,3 \%$. Entre os estudantes de medicina peruanos a taxa de prevalência foi de $24 \%$, mas os autores peruanos acreditam que fatores ambientais, como a situação sociopolítica do país à época do estudo, possam ter influenciado os resultados. No estudo entre estudantes de medicina da Universidade de Salvador, em que se utilizou o MINI Plus, a taxa de prevalência de transtornos depressivos foi de 15,6\%.

Neste estudo não se observaram diferenças significativas entre as taxas de prevalência dos transtornos depressivos nos três períodos dos cursos (início, meio e final). Em estudo longitudinal, Clark e Zeldow (1988) realizaram entrevistas estruturadas com estudantes de medicina em cinco ocasiões. Os resultados apontaram que pelo menos $12 \%$ dos alunos em qualquer período da faculdade apresentavam consideráveis níveis de sintomatologia depressiva, sendo o maior percentual observado no final do segundo ano (25\%). Entretanto não se estimou a prevalência de transtornos depressivos. Em outro estudo observou-se que $76 \%$ dos suicídios entre estudantes de medicina da América do Norte ocorreram nos dois primeiros anos do curso (Rimmer et al., 1982). Os autores postularam que o estresse inerente ao início do curso pode ter contribuído para os resultados. Também nesse estudo não foi determinada a prevalência de transtorno depressivo.

No estudo de Clark e Zeldow (1988), alguns estudantes apresentaram a mesma intensidade de sintomas depressivos nas várias entrevistas realizadas, sugerindo que, para alguns, o humor disfórico era contínuo, e não episódico.

No Brasil, Millan et al. (1995) relataram que o problema do suicídio entre os alunos da Faculdade de Medicina da Universidade de São Paulo (FMUSP) foi uma das motivações que levaram à criação do Grupo de Assistência Psicológica ao Aluno (GRAPAL) nessa faculdade. Os autores fizeram uma investigação retrospectiva acerca do número de suicídios ocorridos entre os alunos da FMUSP no período de 1965 a 1985. A taxa de suicídios encontrada entre os alunos foi aproximadamente quatro vezes maior do que a da população geral.

Nesta pesquisa, a taxa de prevalência para o risco de suicídio foi de $9,6 \%$ do total de alunos entrevistados. Os estudos realizados sobre o tema apontam grande diversidade entre os resultados, provavelmente devido à dificuldade em se qualificar e quantificar com mais precisão o que seria uma ideação suicida.

Salmons e Harrington (1984) identificaram o risco de suicídio em 55\% dos estudantes; Harkavy et al. (1987), em 60\%; e Cordás et al., (1988), em 44\% dos avaliados. Em nosso meio, Miranda e Queiroz (1991), em um estudo realizado na Universidade Federal de Minas Gerais (UFMG), relataram que, em uma amostra de 875 alunos que responderam ao questionário aplicado, $37,5 \%$ dos homens e $36,7 \%$ das mulheres afirmaram já ter pensado em se suicidar, isto é, um total de $37,1 \%$.

Um aspecto importante deste trabalho, e pouco explorado em outros estudos, é a comparação das prevalências de depressão e suicídio entre diversos cursos. As alunas de terapia ocupacional apresentaram 3,6 vezes mais chances de desenvolver depressão maior e 3,7 vezes mais chances de risco de suicídio do que as alunas de medicina e fisioterapia. 
Algumas hipóteses podem ser consideradas. Elias e Murphy (1986) sugerem que "a terapia ocupacional e a promoção da saúde têm muito em comum, usando como exemplo o fato de o enfoque de trabalho dos terapeutas ocupacionais ser 0 desenvolvimento das potencialidades de vida através do aumento das habilidades pessoais, auto-estima e satisfação no viver, que são vistos exatamente como os pré-requisitos para uma vida saudável e de bem-estar".

Certamente, os sedutores objetivos do curso de terapia ocupacional exercem alguma influência na escolha da profissão por parte do aluno, o que poderia se levar a pensar em um processo de seleção, de natureza desconhecida, que justificasse um maior número de pessoas portadoras de transtornos depressivos entre os estudantes do referido curso, tal como foi sugerido por

\section{Referências}

AdewuiaAO, Ola BA,Aloba OO, Mapayi BM, Oginni OO. Depression amongst Nigerian university students: prevalence and sociodemographic correlates. Soc Psychiatry Psychiatr Epidemiol, XX: 1-5, 2006.

Amorim P. Mini International Neuropsychiatric Interview (MINI): validação de entrevista breve para diagnóstico de transtornos mentais. Rev Bras Psiquiatr, 22(3): 106-15, 2000.

Azi L. Transtornos mentais no estudante de medicina. Dissertação. Salvador (BA): Universidade Federal da Bahia; 2003.

Chan WD. Coping with depressed mood among Chinese medical students in Hong Kong. J Affect Disorders, 24: 109-16, 1992.

Clark DC, Zeldow PB. Vicissitudes of depressed mood during four years of medical school. JAMA, 260: 2521-8, 1988.

Cordás TA, Sendacs AM, Gonzales D, et al. Ideação e tentativa de suicídio em uma população de estudantes de medicina. Rev ABP-APAL, 10: 100-2, 1988.

Elias WS, Murphy RJ. The case for health promotion programs containing health care costs: a review of the literature. Am J Occup Therapy, 40(11): 759-63, 1986.

Eric L, Radovanovic Z, Jevremovic I. Mental disorders among Yugoslav medical students. Brit J Psychiatry, 152: 127-9, 1988.

Galli SE, Feijóo LL, Roig RI, Romero ES. Aplicación del "MINI" como orientación diagnóstica en estudiantes de medicina de la Universidad Peruana Cayetano Heredia. Informe preliminar epidemiológico. Rev Med Hered, 13(1): 19-25, 2001.

Giglio JS. Bem-estar emocional em estudantes universitários. Tese. Campinas (SP): Unicamp; 1975.

Hahn MS. Estudo da clientela de um programa de atenção em saúde mental junto ao estudante universitário de São Carlos. Dissertação. Campinas (SP): Unicamp; 1994.

Hahn MS, Ferraz MPT. Características da clientela de um programa de saúde mental para estudantes universitários brasileiros. Rev ABPAPAL, 20(2): 45-53, 1998.
Zoccolillo et al. (1986), em relação a estudantes do curso de medicina nos EUA, diante dos elevados índices de depressão observados entre os acadêmicos naquele país. A hipótese de os alunos de terapia ocupacional terem maior facilidade em comentar aspectos de seu funcionamento psicológico, quando em comparação com os dos cursos de medicina e fisioterapia, também poderia ser um fator diferencial nos resultados observados.

\section{Conclusão}

Neste estudo, a prevalência de transtorno depressivo maior e o risco de suicídio foram significativamente maiores entre os estudantes de terapia ocupacional quando em comparação com os de medicina e fisioterapia.

Harkavy FJM, Asnis GM, Boeck M, Difiore J. Prevalence of specific suicidal behaviors in a high school sample. Am J Psychiatry, 144: 1203-6, 1987.

Lloyd C, Gartrell NK. Psychiatric symptoms in medical students. Comprehensive Psychiatric, 25(6): 552-65, 1984.

Lloyd C. Sex differences in medical students requesting psychiatric intervention. J Nervous and Mental Disease, 171(9): 535-45, 1983.

Millan LR, Barbedo MF. Assistência psicológica ao aluno de medicina: 0 início de uma experiência. Rev Bras Educ Méd, 12(1): 1-40, 1988.

Millan LR, Rossi E, De Marco OLN. A procura espontânea de assistência psicológica pelo estudante de medicina. Rev ABP-APAL, 17(1): 11-6, 1995

Miranda PSC, Queiroz EA. Pensamento suicida e tentativa de suicídio entre estudantes de medicina. Rev. ABP-APAL, 13(4): 157-60, 1991.

Niemi $T$. Use of the mental health care services in Finland by students in different fields of study. J Amer Coll Health, 33: 123-5, 1984.

Nucette LM. Evaluación médico psicológica en estudiantes de medicina. Arch Venezolanos de Psiq y Neurol, 31(65): 51-60, 1985.

Pepitone-Arreola-Rockwell F, Rockwell D, Core N. Fifty two medical student suicides. Am J Psychiatry, 138: 198-201, 1981.

Rimmer J, Halikas JA, Shuckit MA. Prevalence and incidence of psychiatric illness in college students: a four year prospective study. J Amer Coll Health, 30: 207-11, 1982.

Ross M. Suicide among physicians a psychological study. Dis Nerv Syst, 31: $145-50,1973$

Salmons PH, Harrington R. Suicidal ideation in university students and other groups. Int J Soc Psychiatry, 30: 201-5, 1984.

Segal BE. Epidemiology of emotional disturbance among college undergraduates: a review and analysis. J Nervous and Mental Disease, 143: 348-62, 1966.

Zoccolillo M, Murphy GE, Wetzel RD. Depression among medical students. J Affect Disorders, 11: 91-6, 1986. 